\title{
When is enough, enough? An Examination of Student Engagement when Watching Online Group Project Presentations
}

\author{
Manasvi Kumar \\ University of Arizona \\ manasvik@email.arizona.edu
}

\author{
Joseph S. Valacich \\ University of Arizona \\ valacich@email.arizona.edu \\ David Kim \\ University of Arizona \\ davidkim7@email.arizona.edu
}

\author{
Jeffrey L. Jenkins \\ Brigham Young University \\ jjenkins@byu.edu
}

\begin{abstract}
In traditional face-to-face classes, conventional wisdom suggests that delivering and watching group project presentations is a valuable learning experience. In this research, we examine the limits of student engagement and learning in an asynchronous online context. Specifically, 249 undergraduate students were assigned to perform peer evaluations of multiple tenminute project presentations. The online learning platform collected objective viewing behavior for each student, allowing us to use viewing time as a proxy for engagement. We also collected self-reported attitudes towards the assignment, finding that while students value providing feedback, they do not consider it a valuable use of their time. Students who engage more are also likely to receive a better final course grade. Finally, students exhibit different types of viewing behavior (i.e., personas) when evaluating multiple videos. Based on these results, we provide suggestions for improving the design of online group presentation and peer-review assignments.
\end{abstract}

\section{Introduction}

The global pandemic involving the novel Coronavirus in 2019 (i.e., COVID-19) has entirely changed the way we live, work, and learn. Severe restrictions (e.g., social distancing) and lockdown measures forced people to work remotely, engage in distance education, and collaborate online. For instance, in March 2020, World Bank Education reported that more than 680 million students lived in countries with fully closed schools, while only 92 million students lived in countries that partially closed schools [1]. A case study that examined campus traffic and online learning during the COVID-19 pandemic also suggested that the volume of virtual classes grew rapidly as lockdown measures were imposed [2].

The rapid development in information technologies (e.g., video and audio-conferencing tools), along with improved network bandwidth to homes, allowed educational institutions and students to shift to distance education. This shift was not entirely smooth, as replicating specific class activities and assignments remains challenging [3]. For instance, individual or group presentations are considered a critical learning experience but are difficult to include as a part of a distance learning course [4]. Instructors can choose to replace individual or group presentations with another assignment, but it may negatively impact the learning experience. Including an individual or group presentation is a common part of many course design recommendations in various computing disciplines (e.g., ACM Computing Curricula 2020) [5,6], given that effectively presenting technical information is a highly valued competency in many organizational settings [7]. In addition to pure computing class contexts, Du, Johnson, and Keil (2004) surveyed 206 institutions to evaluate the curriculum content related to project management, finding that most programs require project presentations for both groups and individuals [8]. Venkatesh (2008) suggests the need for MBA students to design and deliver presentations to appreciate the need for different competitive strategies in different organizational environments and when students learn in multi-course integrated MBA programs $[9,10]$.

A major issue with including individual or group presentations in distance learning courses is that it is difficult to gauge other students' participation and engagement. In an in-person class, the instructor or facilitator can observe listeners' behaviors to determine whether they are engaged with the presented material. Assessing student engagement in an online setting is 
different and difficult. For synchronous classes (i.e., live-online), it is not easy for the instructor to review the presented materials and facilitate the audience's engagement. For asynchronous classes (i.e., presentation materials uploaded to a cloud server), the audiences' participation and engagement could only be assessed by collecting feedback (i.e., students' actual viewing behavioral cannot be easily assessed). These issues are further exacerbated when instructors require multiple presentations to be assessed which could take an exorbitant amount of time to complete.

In this paper, we explore the relationships between student perceptions on presentation evaluations, their engagement behavior while completing the task, along with their overall class performance. We find that students are more likely to agree with the importance of providing feedback to other students, but do not perceive it to be a valuable use of their time. We also find that students' actual viewing behavior is a proxy for students' mastery with the course material (i.e., their final course grade). These findings are consistent with conventional wisdom and previous research which demonstrates a strong relationship between students' online behavior (e.g., seconds spending viewing postings, number of postings viewed, etc.) and their final grades [11]. Finally, we find that students engage in different types of viewing behavior (i.e., personas) as they complete multiple evaluations. Based on the results obtained, we provide several suggestions to help instructors improve course design to ensure that a majority of the students maintain acceptable levels of engagement while performing evaluations.

\section{Methodology}

We designed a study to understand the limits of student engagement when evaluating online presentations. Students enrolled in an undergraduate operations management class at a public university in the US were considered for the study. As part of the course requirement, all students were part of a fivemember project team which submitted a 10-minute online recorded presentation about a case-study. They were also required to individually evaluate five randomly assigned presentations. We asked the students to complete an online survey where they self-report how providing feedback to other students about their presentations was a valuable use of their time and improves their learning experience. They also selfreport their perceived importance of student feedback in such assignments. As the presentations were hosted on an online learning platform that records which files are opened and for how long, we capture file access and opening duration as a proxy measure for actual engagement. Thus, this study utilizes not only self- reported measures like attitudes toward learning, value of time, and importance of the task but also actual engagement while completing the evaluations. Utilizing these diverse data sources helps us develop a holistic understanding of how students perceive and go about completing the task of online presentation evaluations.

\subsection{Survey Design}

The survey primarily consists of two types of questions: demographic questions and questions capturing student attitudes towards the evaluation assignment. At the start of the survey, students provide answers to several demographic questions. They then provide answers to questions concerning whether the evaluation assignment was a valuable use of their time i.e., "The feedback assignment was a good use of my time", provided a useful learning experience i.e., "The feedback assignment was a valuable learning experience" and is an important task i.e., "It is important for me to provide feedback to other students." Responses to these questions were calibrated on a 5point Likert scale, from "Strongly Disagree" to "Strongly Agree." Students were then allowed to provide consent for the use of their survey responses and educational records for the class (i.e., their class performance in the course used in the study). Those who completed the survey (irrespective of their choice to provide consent) received 5 points of extra credit when calculating their overall class performance.

\subsection{Data Preprocessing}

Of the 420 students enrolled in the class, 365 students completed the survey (86.9\%). 289 of them agreed to allow the use of their educational records pertaining to the course for the purpose of this study (i.e., we had to gain approval from the Institutional Review Board [IRB] and comply with FERPA guidelines). We analyzed the viewing times of these 289 students and found that several students viewed individual videos for greater than 20 minutes, with the maximum individual time being 145 minutes for a single video. As students had worked on creating their own video prior to evaluation, they are clearly familiar with the content, and it is unlikely that a student watched a video more than two times while evaluating it. We therefore exclude cases where the viewing time exceeded 20 minutes (twice the length of any video). After preprocessing, records from 249 students were used to perform the analysis.

While collecting viewing data from the online learning platform, we have access to a students' last date of visit, total time spent, and the number of visits to the page corresponding to a video. Thus, we are unable to 
capture the sequence in which students may have viewed each video. We negate this issue by sorting students' viewing times of individual videos in descending order, from longest to the shortest, as it is most likely and logical that viewing time would decline as a person watches subsequent videos. In other words, we assume that students got experientially faster in evaluating presentations and the sorted order will be similar to the true order of viewing.

In addition to the individual view times of videos, we include survey responses and final letter grades obtained by the students in the class in our analysis. We consider final letter grades as opposed to grades on the group presentation project as it is a better representation of the students' individual understanding of the course, while the latter reflects the grade of the entire group which created the presentation.

\section{Results}

In this section, we summarize results of survey responses to identify perceived student attitudes towards various facets of the assignment. As perceived behaviors often differ from actual behavior, we also analyze viewing times of various videos as a proxy for engagement. The analysis of viewing times was performed from two perspectives - Aggregate and Individual. From an aggregate perspective, we answer various questions such as:

- How much time do students spend on the assignment?"

- $\quad$ "How much time do students spend on evaluating each video?" and

- "How many videos do students evaluate for a given amount of time?"

Answering these questions helps capture overall actual student engagement towards the assignment. We then attempt to identify individual viewing behaviors among students and generate individual "viewing personas" to help distinguish them. These personas correspond to varied strategies used by the students to complete the assignment. Finally, we analyze how various aggregate behaviors and individual personas influence survey responses and performance in the class. This helps us test conventional wisdom that students who spend adequate time to complete their assignments receive high grades.

\subsection{Survey Results}

As mentioned earlier, students answer several questions pertaining to the value of time, learning outcomes and importance of feedback during the survey. Each response is recorded on a 5-point Likert scale and is recalibrated on a scale of 1 to 5 , with 1 corresponding to Strongly Disagree and 5 to Strongly Agree. Statements worded negatively are scaled appropriately to ensure that participants expressing a positive attitude (i.e., assignment was a good use of time, good learning experience and providing feedback is important) receive a greater score than those who express a negative attitude. For each student, we estimate his/her corresponding attitude by computing the average of all scores on relevant questions. Table 1 summarizes the results for the survey responses to various questions asked during the survey.

Table 1. Summary statistics of survey responses

\begin{tabular}{|c|c|c|c|c|}
\hline Attitude & Min. & Max. & Median & Average \\
\hline Time & 1 & 5 & 3 & 3.03 \\
\hline Learn & 1 & 5 & 3.33 & 3.22 \\
\hline Feedback & 1.33 & 5 & 3.67 & 3.46 \\
\hline
\end{tabular}

As indicated in Table 1, the average score of student attitudes corresponding to the assignment being a valuable use of their time is 3.03 , and 3.22 when viewed as a valuable learning experience (i.e., using a 5-point scale with 3 being neutral). The average score of student attitude is highest, 3.46, when asked about their views on student feedback. Thus, most students agree that providing feedback is important but do not necessarily see the feedback assignment as a valuable use of their time. It is possible that most students think that viewing 5 videos for 10 minutes each to provide feedback may be excessive. We now analyze the actual viewing times of these videos.

\subsection{Aggregate Viewing Behaviors}

Before analyzing view times of individual videos, we analyze view time at the assignment level. We first answer the question: "How much time do students spend on the assignment?" To do this, we aggregate the view times of all five videos for each student and analyze the distribution. Ideally, each student is expected to have viewed all 5 videos at least once for a total viewing time of 3000 seconds (i.e., ideal viewing time: 5 videos X 10 minutes X 60 seconds $=3000$ seconds). Figure 1 reveals the histogram for aggregate view times, with those less than 1500 seconds ( $50 \%$ of ideal time) highlighted in red and those greater than 3000 seconds (ideal time) highlighted in blue. Visually, it is clear that a significant part of the class spent less than half of the ideal time on the assignment. The distribution has an average time of 1499 seconds (15 minutes or $\sim 50 \%$ of the ideal time) while the median is 1293 seconds ( 12.5 minutes or $43.1 \%$ of ideal time). This is consistent with the results on the survey responses, where students indicate that 
providing feedback is not a very valuable use of their time. There are also 28 students $(11.24 \%)$ who spend more than the ideal time on the assignment. A pairwise t-test conducted on all these distributions suggest that the viewing times for these videos are different $(\mathrm{p}<0.01)$.

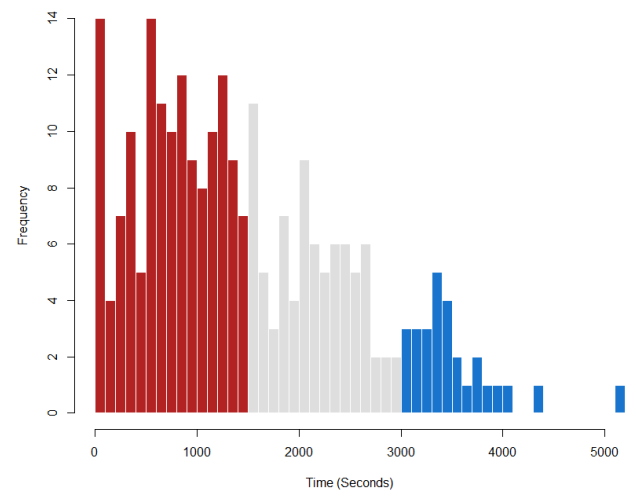

Figure 1. Histogram of aggregate view time

We now analyze view times at a video level. Specifically, we answer the question: "How much time do students spend on evaluating each video?” As 249 students were each asked to view 5 videos, there were a total of 1245 (i.e., 249 students X 5 videos = 1245) evaluations that were completed. The average view time for an individual video is 299 seconds ( $~ 5$ minutes) while the median time is 226 seconds ( 3.77 minutes). The distribution of the number of evaluations completed after viewing for less than a specified amount of time is shown in Table 2. More than half of the responses generated (642 or $52 \%$ ) received less than 4 minutes of view time. This indicates that more than half of the responses generated involved students either skimming through or skipping the contents of the video. Notably, 191 responses $(15 \%)$ were generated after viewing the video for less than a minute.

Table 2. Cumulative distribution of responses based on view time

\begin{tabular}{|c|c|c|}
\hline $\begin{array}{c}\text { Maximum } \\
\text { minutes viewed }\end{array}$ & $\begin{array}{c}\text { Number of } \\
\text { responses }\end{array}$ & $\begin{array}{c}\text { Percentage of } \\
\text { responses }\end{array}$ \\
\hline 1 & 191 & 15 \\
\hline 2 & 361 & 29 \\
\hline 3 & 518 & 42 \\
\hline 4 & 642 & 52 \\
\hline 5 & 743 & 60 \\
\hline 6 & 835 & 67 \\
\hline 7 & 914 & 73 \\
\hline 8 & 958 & 77 \\
\hline 9 & 1016 & 82 \\
\hline 10 & 1076 & 86 \\
\hline
\end{tabular}

We now answer the question: "How many videos do students evaluate for a given amount of time?" For each student, we have a list of 5 individual view times (corresponding to the 5 videos they were required to evaluate). As in Table 2, we partition the viewing behavior at minute intervals, from 1 to 10 . We then compute the number of students who viewed at least 1 , $2,3,4$ or all 5 videos for the required amount of time. Table 3 summarizes the results of this analysis. From Table 3, it is evident that more than half the class $(n=125, \%=50.2)$ observed at least one video for greater than 7 minutes. This indicates that the most watched video for around half of the class is more than 7 minutes long. In contrast, around half of the class $(n=123$, $\%=49.4$ ) observed all 5 videos for greater than 2 minutes. This indicates that for around half of the class, the least viewed video is less than 2 minutes long. The data suggests that there is a significant difference between the most watched and least watched video for most students.

Table 3. Cumulative distribution of participants based on view time and number of videos

\begin{tabular}{|c|c|c|c|c|c|}
\hline \multirow{2}{*}{ Minutes } & \multicolumn{5}{|c|}{ Videos } \\
\cline { 2 - 6 } & $>=1$ & $>=2$ & $>=3$ & $>=4$ & 5 \\
\hline$>=1$ & 232 & 229 & 219 & 209 & 171 \\
\hline$>=2$ & 221 & 206 & 182 & 156 & $\mathbf{1 2 3}$ \\
\hline$>=3$ & 202 & 175 & 144 & 118 & 89 \\
\hline$>=4$ & 181 & 147 & 116 & 96 & 65 \\
\hline$>=5$ & 164 & 122 & 94 & 74 & 48 \\
\hline$>=6$ & 147 & 101 & 72 & 55 & 37 \\
\hline$>=7$ & $\mathbf{1 2 5}$ & 84 & 55 & 40 & 28 \\
\hline$>=8$ & 119 & 69 & 46 & 34 & 19 \\
\hline$>=9$ & 100 & 58 & 36 & 23 & 13 \\
\hline$>=10$ & 82 & 45 & 25 & 15 & 4 \\
\hline
\end{tabular}

\subsection{Individual Viewing Personas}

We now analyze the viewing times of individual videos. As mentioned earlier, the exact sequence in which each student viewed the videos cannot be known due to limitations in the data collection and reporting capabilities of the online learning platform. We therefore sort videos based on view time and analyze them accordingly. Table 4 provides summary statistics for view times of these 5 videos. The visualization of these distributions is shown in Figure 2. 
Table 4. Summary statistics of individual view times

\begin{tabular}{|c|c|c|c|c|c|}
\hline & Min. & Max. & Med. & Avg. & S.D. \\
\hline Video 1 & 0 & 1187 & 422 & 479 & 311 \\
\hline Video 2 & 0 & 1120 & 298 & 349 & 249 \\
\hline Video 3 & 0 & 1028 & 215 & 275 & 210 \\
\hline Video 4 & 0 & 1000 & 169 & 228 & 191 \\
\hline Video 5 & 0 & 820 & 117 & 169 & 170 \\
\hline
\end{tabular}

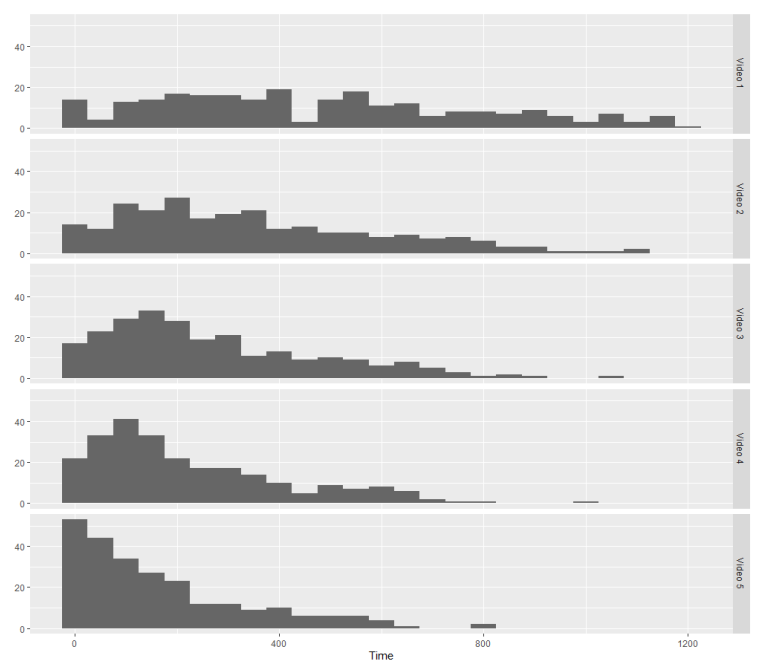

Figure 2. Histograms of individual video view times

Table 4 indicates that the average time taken by a student to view the most watched video (Video $1-478.8$ seconds) is 2.8 times that of the least watched video (Video 5 - 168.6 seconds). The average and median time goes down. Their distributions are also different, as suggested in Figure 2. A pairwise t-test conducted on all these distributions (1 through 5) suggest that the viewing behaviors for these videos are different $(p<0.05)$. This evidence suggests that on average, students' viewing behavior changes as he/she evaluates the 5 videos. We now attempt to categorize these viewing behaviors based on our observations.

We earlier observed that 191 of the 1245 evaluations were completed in less than a minute (see Row 1 in Table 2). As the presentation is based off a case study that is challenging at an undergraduate level, we posit that it is very difficult to provide reasonable feedback upon such limited viewing. Any student who completes his/her evaluation by viewing at least one video for less than a minute has likely not done so honestly. We therefore classify such students who viewed at least one video for less than a minute as "Cheaters."

On the other end of the spectrum, we would also like to classify those students who performed the assignment diligently. One way to identify these individuals would be to identify all individuals who viewed all five videos for their entire duration (10 minutes). While this is a reasonable criterion, Table 4 indicates that the average time taken to formulate a response for the least watched video is less than 3 minutes. Setting the threshold to be 10 minutes seems unreasonable, and also discounts the potential experiential effect a student may gain as he/she completes the assignment and gradually gets more efficient and faster. Table 2 suggests that more than half of all formulated responses took less than 4 minutes to complete. As the conscientious student is expected to provide a thorough response on all videos, we anticipate that these students spend greater than average time on their responses compared to others. We thus define those students who view all their videos for greater than or equal to 5 minutes as "Conscientious" students.

Between the two ends of the spectrum of viewing behavior - i.e., from the "Conscientious" student to the "Cheater" student - lie another class of students who don't cheat (i.e., view a video for less than a minute but also do not spend as much time on formulating responses as the "Conscientious" students), that we categorize as "Satisficing" students. Satisficing students do not necessarily try to provide the best feedback but a satisfactory one, spending greater than 1 minute but less than 5 minutes on all their reviewed videos.

An interesting result obtained while analyzing overall individual viewing times relates to the difference between the times of the most and least watched videos. Specifically, the view time of the most watched video is roughly 2.8 times that of the least watched video. There are clearly important differences in student viewing behaviors on their most and least watched videos and it is important to classify these participants carefully. Our current classification system captures some of these participants. For instance, a student classified as a "Cheater" may have watched Video 1 for 1.5 minutes and Video 5 for 0.5 minutes; a 66\% drop-off in viewing behavior. A "Conscientious" student may have watched Video 1 for 15 minutes and Video 5 for 5 minutes; again a drop-off of 66\%. Finally, a "Satisficing" student may have watched Video 1 for 4.5 minutes and Video 5 for 1.5 minutes maintaining a drop-off of $66 \%$. In these instances, while there is a significant drop-off, there are other factors which better define the students' viewing behavior.

It is possible however, for students to have such significant drop-offs without being categorized in the previously mentioned categories. Specifically, it is possible for a student to have watched Video 1 for 6 minutes and Video 5 for 2 minutes (drop-off is 66\%) and not be classified based on the categories defined thus far. It is unlikely that the difference in view times could 
be attributed to the video, as no presentations received were of such extreme superior/inferior quality to warrant such differences in view times. It is however, likely that the student viewed one video for a long time (due to distraction or frustration) and since then exhibit a change in viewing behavior in the latter videos. We classify those students who are not "Cheaters", "Conscientious" or "Satisficing" but spend more than three times the amount of time of their least watched video on their most watched video as "Drop-off" students, owing to the significant drop-off observed in their view times.

It is possible for students to exist with view times such that they do not fall into the aforementioned classes. They may not have provided unsatisfactory responses as "Cheaters" but have also not performed as diligently as the "Conscientious" students. Not all their responses involve satisficing behavior but they also do not exhibit extreme drop-offs in viewing time. These students have spent typical time on all videos and have likely become faster owing to experiential effects. We define these students who do not belong to any of the aforementioned categories as "Conventional" students as they exhibit expected behavior while completing the evaluations. Table 5 summarizes the various classification of students and their definitions.

Table 5. Viewing behavior-based personas and their characteristics

\begin{tabular}{|c|c|}
\hline Respondent & Viewing Characteristics \\
\hline Cheater & Video 5 for $<1 \mathrm{~min}$ \\
\hline Conscientious & Video 5 for $>5 \mathrm{mins}$ \\
\hline Satisficer & Video 1 for $<5 \mathrm{~min}$ \\
& Video 5 for $>1 \mathrm{~min}$ \\
\hline Drop-off & Video 1 for $>5 \mathrm{mins}$ \\
& $\begin{array}{c}\text { Video } 5 \text { for }>1 \text { min and }<5 \text { mins } \\
\text { Video } 5 / \text { Video } 1>=3\end{array}$ \\
\hline Conventional & Video 1 for $>5$ mins \\
& $\begin{array}{r}\text { Video } 5 \text { for }>1 \text { min and }<5 \text { mins } \\
\text { Video } 5 / \text { Video } 1<3\end{array}$ \\
\hline
\end{tabular}

Figure 3 provides a line chart of the average times (with standard error) taken by the different personas when completing the assignment. There are several interesting observations from this analysis. First, the "Conscientious" student spends on average greater than 10 minutes on two of the five videos. This is in stark contrast to other class of students. Second, the "Cheater" student spends, on average, more time on their most viewed video than does the "Satisficer" student. This suggests that that the viewing behavior "Satisficer"'s strategy to provide a satisfactory response remains the same while completing the assignment but the "Cheater" is likely to have had a change of strategy. Third, a much greater change of strategy is observed in the "Dropoff" student, who spends on average greater than 10 minutes on the most watched video but less than 3 minutes on the least watched video. The distribution of the generated personas is tabulated in Table 6. The largest proportion of students belong to the "Cheaters" category $(31.7 \%)$, while "Satisficers" constitute only $12.4 \%$ of students who completed the survey.

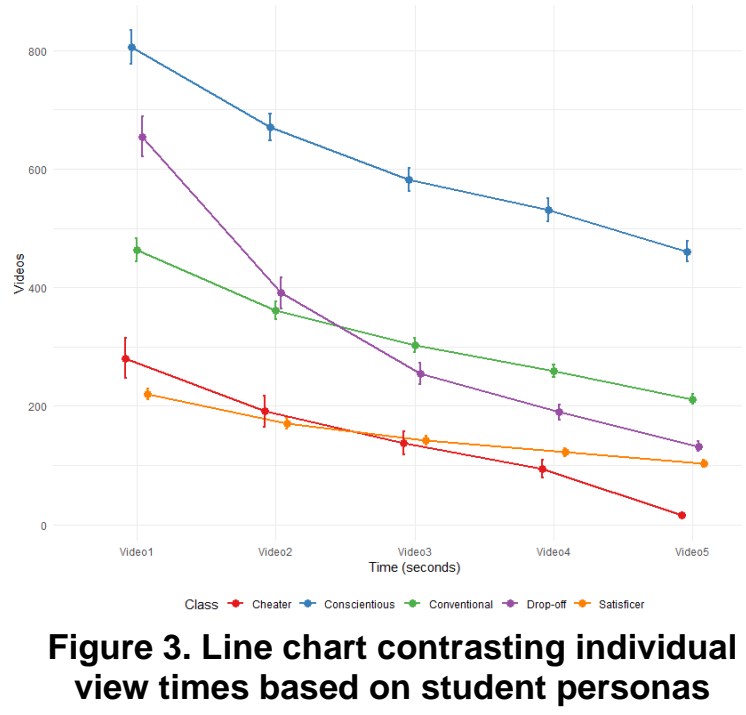

Table 6. Distribution of personas

\begin{tabular}{|c|c|c|}
\hline Persona & Number & Percentage \\
\hline Cheater & 79 & 31.7 \\
\hline Conscientious & 48 & 19.3 \\
\hline Satisficer & 31 & 12.4 \\
\hline Drop-off & 49 & 19.7 \\
\hline Conventional & 42 & 16.9 \\
\hline
\end{tabular}

According to our proposed definition of a "Cheater", a student who provides feedback after viewing even a single video for less than a minute qualifies to be a "Cheater". As this is applicable to roughly a third of the sample, we examine this group further by tabulating the number of students who viewed exactly $1,2,3,4$ or 5 videos for less than a minute (Table $7)$. We found that $37(46.8 \%)$ of "Cheaters" viewed exactly 1 video for less than a minute while $17(21.5 \%)$ of them viewed all 5 videos for less than a minute. While there is considerable variability in the degree of cheating among the "Cheater" class, we perform the rest of our analysis retaining the original definition. 
Table 7. Distribution of participants with $<1$ minute view time

\begin{tabular}{|c|c|}
\hline \# videos with < 1 min & \# of Participants \\
\hline 1 & 37 \\
\hline 2 & 11 \\
\hline 3 & 9 \\
\hline 4 & 5 \\
\hline 5 & 17 \\
\hline
\end{tabular}

\subsection{Effect of view times on survey responses}

In this section, we compare view times to actual survey responses. Based on time taken to complete the assignment, we saw earlier that the majority of students took less than 25 minutes (half the ideal time) to complete the assignment, while a minority took greater than 50 minutes (ideal time) to complete it. Table 8 summarizes the average scores of these groups of students on the questions pertaining to the student attitudes questions. It is observed that students who spend less than 25 minutes on the assignment later reported that the assignment was not a valuable use of their time and was not a good learning experience (compared to the other groups). They also comparatively report lower values on the importance of providing feedback.

Table 8. Contrasting responses based on aggregate view time

\begin{tabular}{|c|c|c|c|}
\hline $\begin{array}{c}\text { Aggregate } \\
\text { time }\end{array}$ & Time & Learn & Feedback \\
\hline$<25$ mins & 2.894 & 3.106 & 3.333 \\
\hline $25-50 \mathrm{mins}$ & 3.209 & 3.295 & 3.679 \\
\hline$>50 \mathrm{mins}$ & 3.179 & 3.631 & 3.512 \\
\hline
\end{tabular}

We also found that several students follow different viewing behaviors while completing the assignment (Table 5). Comparing their viewing behaviors to survey responses (Table 9) reveals that students classified as "Satisficers" found the assignment to be the least valuable use of their time while those who were classified as "Conscientious" found it to be the most valuable. "Conscientious" students also found the assignment to be a valuable learning experience while the students experiencing "Dropoff" least found it so. Scores on the importance of feedback saw relatively higher scores from all groups of students.
Table 9. Contrasting responses based on viewing-based personas

\begin{tabular}{|c|c|c|c|}
\hline Persona & Time & Learn & Feedback \\
\hline Cheater & 2.962 & 3.110 & 3.464 \\
\hline Conscientious & 3.219 & 3.521 & 3.493 \\
\hline Satisficer & 2.823 & 3.280 & 3.355 \\
\hline Drop-off & 3.0 & 3.102 & 3.599 \\
\hline Conventional & 3.107 & 3.206 & 3.349 \\
\hline
\end{tabular}

\subsection{Effect of view times and survey responses on Class Performance:}

As mentioned earlier, the students who completed the survey consented to provide access to education records pertaining to the course. We found that 140 (56.2\%) out of the 249 students who completed the survey got an A grade on the course. In this section, we compare the various view times, personas created and survey responses to the final grade obtained by the student for the course. Figures 4 and 5 demonstrate how overall grade is affected by aggregate and individual view times on the assignment. At an aggregate level, we observe that students who spend more than half the ideal time in providing feedback are more likely to receive an A grade than those who don't. At an individual video level, we observe that students who receive an A grade spend on average more time on every video (from most watched to least watched) as opposed to those who do not.

We now compare the individual viewing behaviors on the assignment to class performance. Figure 6 displays the proportion of students who receive an A grade for every viewing behavior. We observe that students classified as "Cheaters", "Satisficers" or "Drop-offs" all have a similar and less than average (56.2\%) chance of receiving an A grade on the course. This observation is in coherence with conventional wisdom that students who do not perform their tasks diligently and look for shortcuts are likely to do worse than those who do not. Students who are classified as "Conscientious" or "Conventional" are more likely to receive an A grade, with the latter being more likely than the former. This result goes against conventional wisdom, but may be interpreted as follows: Students who repeat certain sections of the video (thereby increasing view time) may do so out of wanting to be thorough or because they do not grasp all concepts covered in the video in one viewing. They report finding the assignment to be a valuable use of their time and a useful learning experience (Table 9). It is likely that these students benefit the most from the assignment and despite not performing as well as the "Conventional" students, easily outperform the rest of the class. Finally, Table 10 distinguishes the average scores on the survey 
based on class performance. Students who received an A grade report higher scores on the importance of student feedback, value for time, and useful learning experience than those who do not.

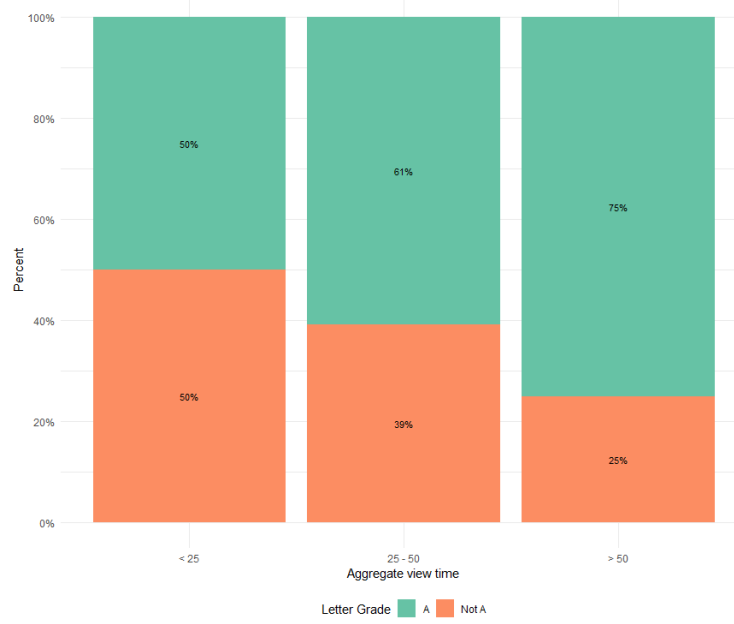

Figure 4. Percentage bar chart contrasting letter grades based on aggregate view time

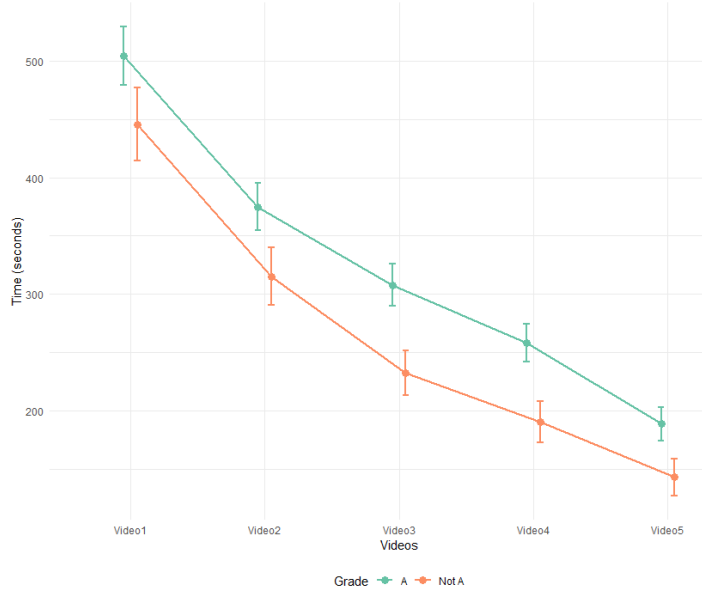

Figure 5. Line chart contrasting individual video view times based on letter grade

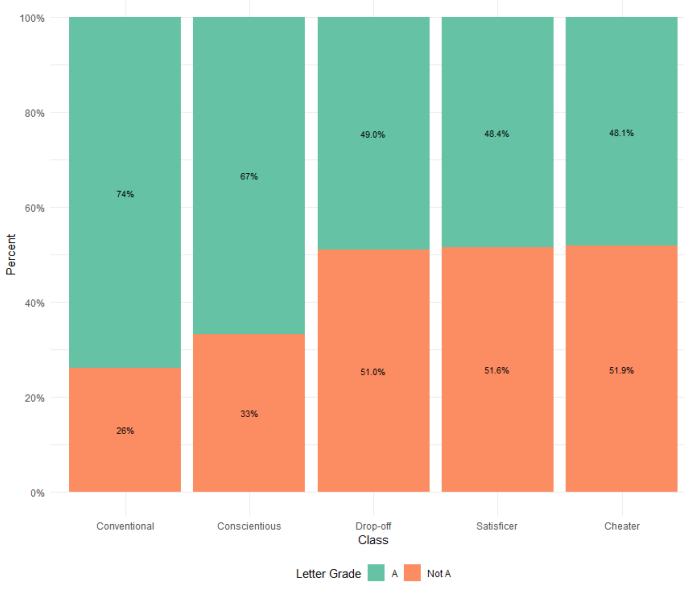

Figure 6. Percentage bar chart contrasting letter grades based on student personas

Table 10. Contrasting responses based on grade on the course

\begin{tabular}{|c|c|c|c|}
\hline Grade & Time & Learn & Feedback \\
\hline A & 3.075 & 3.310 & 3.476 \\
\hline Not A & 2.963 & 3.116 & 3.446 \\
\hline
\end{tabular}

\section{Discussion}

In this paper, we utilize survey data, actual view time data, and course grade data to explore the relationships between student perceptions on presentation evaluations, engagement behavior during task completion, and overall class performance. The survey results obtained suggest that students agree that providing feedback is important but did not see the task of providing feedback as a valuable use of their time (Table 1). Analyzing individual and aggregate view times reveals that several students do not watch all 5 videos entirely prior to providing feedback (Table 2 and 3 ). Together, these results suggest that while the students value providing feedback, the task design to collect student feedback is probably not optimal and therefore students aren't spending the ideal amount of time in providing feedback.

To overcome this issue, we suggest that reducing the overall ideal time requirement to complete the task could be beneficial. One way to do so could be to reduce the number of presentations a student evaluates. Currently, each of the 420 enrolled students evaluates 5 presentations. The overall class has 84 unique presentations (420 students in a 5-member team). Therefore, each presentation is evaluated a total of 25 times ((420 X 5) / 84), which seems excessive. Considering that less than half the class (123) views all 5 videos for greater than 2 minutes (Table 3), reducing 
the number of evaluations per student also seems necessary. Another approach could be to reduce the overall length of the presentation. Table 2 suggests that more than half the feedback generated was less than 4 minutes. The optimal approach would involve a combination of the two suggestions - reduce the number of videos and the length of the video. Table 3 suggests that almost half the class viewed at least 3 videos for at least 4 minutes. Therefore, a good starting point could be to redesign the assignment such that each student evaluates 3 presentations with each presentation being no longer than 5 minutes each.

We also found that students exhibit different viewing behaviors as they evaluate the presentations (Table 5). Some students engage in unethical behaviors (Cheaters), while some provide suboptimal feedback (Satisficers) and others reduce engagement after initial ideal behavior (Drop-offs). All these students perform worse than average on the final course grade (Figure 6). There are also students who do not engage in the aforementioned behaviors (Conventional) or those who spend greater than average time on all their evaluations (Conscientious). These students tend to perform better than average, as is consistent with conventional wisdom that students who spend adequate time on completing the assignment receive better grades. We found that the "Conscientious" student performs worse than the "Conventional" student. This indicates that the students currently classified as "Conscientious" may also include students who gave an honest attempt but struggled through the assignment. It is difficult to identify such students through engagement behavior alone, and alternative approaches are required to help such students improve their understanding of course material. Finally, we also report that view times alone are also useful indicators of a students' understanding and therefore their final grade. Students who receive an A grade spend more time than those who didn't on all 5 videos.

We posit that instructors of all courses which require online presentation evaluations can benefit greatly from the results of this paper. This is especially the case if the presentations are hosted in an online learning platform (or other platforms) which enable them to monitor page access times. Performing an analysis based on view times similar to the one completed in this paper would greatly help instructors gauge student engagement and find the optimal assignment design to maximize student engagement. Feedback received through online surveys could help assess whether students learn by providing feedback, find it a good use of their time, and value providing it in the first place. Repeating these steps over consecutive semesters as the assignment is fine-tuned based on these diverse data sources could help identify an optimal design of feedback presentations in a particular context.

\section{Limitations}

In this paper, we approximate video view times as a proxy measure for engagement. We derive these view times from the online learning platform that captures total time spent on the page by the user. One limitation of this approach is the assumption that the student spent the entirety of the time on the page viewing the video in an engaged manner. While we account for obvious instances of unengaged behavior by discarding observations which are greater than 20 minutes in length, it is likely that students may not have been fully engaged in viewing the video while on the page. Another limitation in this study is that we cannot capture the sequence in which videos were viewed. This limitation occurs because of the granularity at which the last viewed instance is reported in the online learning platform (at a day level). We performed our analysis under the assumption that most students will get experientially faster when evaluating presentations and the sorted order will be similar to the true order of viewing. This may not be true in a variety of circumstances. We also assumed that all presentations are similar in quality and require no more than one viewing by every student to perform a suitable evaluation. While this may be true for most instances, it is conceivable that some videos may require additional viewing by certain students. Finally, the trends observed and discussed in our results are not always statistically significant. This is in part, due to the sampling issues we faced during the data collection process (Only 249 out of potentially 421 data points were useful).

\section{Conclusion}

In this paper, we designed a study to understand the limits of student engagement while evaluating online presentations. Students who were required to provide feedback to online presentations were recruited for the study. We utilized self-reported measures like attitudes toward learning, value of time, and importance of the task as well as actual engagement while completing the evaluations to perform analysis. We explored the relationships between student perceptions on presentation evaluations, their engagement behavior while completing the task, and class performance. We found that students' behavioral data when evaluating online presentations indicates students' engagement with the course material (i.e., their final course grade). We also found that students engage in different types of viewing behavior as they completed multiple evaluations. We encourage instructors to perform similar analyses (as applicable) to better gauge student engagement in the context of online presentations and help improve their course design to ensure acceptable 
levels of students' engagement while performing evaluations.

\section{Acknowledgment}

We would like to thank Dr. Eyran Gisches (University of Arizona) for his support and guidance during the data collection process for the study.

\section{References}

[1] World Bank Education, "World Bank Education COVID19 School Closures Map", World Bank. https://www.worldbank.org/en/data/interactive/2020/03/24/w orld-bank-education-and-covid-19

[2] Favale, T., F. Soro, M. Trevisan, I. Drago, and M. Mellia, "Campus traffic and e-Learning during COVID-19 pandemic", Computer Networks 176, 2020, pp. 107290107290.

[3] Miyagawa, S., and M. Perdue, "A Renewed Focus on the Practice of Teaching", 2020 https://www.insidehighered.com/advice/2020/11/11/switchin g-online-teaching-during-pandemic-may-fundamentallychange-how-faculty

[4] Sojayapan, C., and J. Khlaisang, "The effect of a flipped classroom with online group investigation on students' team learning ability”, Kasetsart Journal of Social Sciences, 2018.
[5] Associations for Computing Machinery (ACM), "Curricula Recommendations", https://www.acm.org/education/curricula-recommendations, Computing Curricula 2020.

[6] Gorgone, J., P. Gray, D.L. Feinstein, et al., "MSIS 2000: Model Curriculum and Guidelines for Graduate Degree Programs in Information Systems", Communications of the Association for Information Systems 3, 2000.

[7] Lee, A., "Effective Use of PowerPoint in Professional \& Technical Presentations", Writing Commons. https://writingcommons.org/article/effective-use-ofpowerpoint-in-professional-technical-presentations/

[8] Du, S., R. Johnson, and M. Keil, "Project Management Courses in IS Graduate Programs: What is Being Taught?", Journal of Information Systems Education 15, 2004.

[9] Slater, J.S., D.J. McCubbrey, and R.A. Scudder, "Inside an integrated MBA: An information systems view", Mis Quarterly, 1995, pp. 391-410.

[10] Venkatesh, V., "Teaching Tip: 'One-Size-Does-Not-FitAll': Teaching MBA Students Different ERP Implementation Strategies", Journal of information Systems Education 19, 2008, pp. 141-146.

[11] Morris, L.V., C. Finnegan, and S.-S. Wu, "Tracking student behavior, persistence, and achievement in online courses", The Internet and Higher Education 8(3), 2005, pp. 221-231. 